\title{
DETERMINAÇÃO DO MOMENTO DE COLHEITA DA Brachiaria brizantha (Hochest.) Stapf. cv. MARANDU PARA PRODUÇAÕ DE SILAGEM
}

\author{
Determination of the harvest moment of Brachiaria brizantha $c v$. Marandu for ensilage prodution
}

\author{
Cristiano Gonzaga Jayme ${ }^{1}$, Lívio Ribeiro Molina ${ }^{2}$, Lúcio Carlos Gonçalves ${ }^{3}$, \\ Diogo Gonzaga Jayme ${ }^{4}$, Daniel Ananias de Assis Pires 5 , Iran Borges ${ }^{6}$
}

\begin{abstract}
RESUMO
Conduziu-se este trabalho, com o objetivo de determinar o momento de colheita da Brachiaria brizantha para produção de silagem. As silagens foram abertas após 56 dias de fermentação. Os tratamentos utilizados foram quatro idades de crescimento: 28,56 , 84 e 112 dias. Foi utilizado delineamento inteiramente casualisado, com quatro repetições por tratamento. As produções de MS aumentaram com o avanço da maturidade da planta, variando de 3,04 a 11,47 t/ha. O menor teor de MS entre as silagens ocorreu aos 28 dias $17,16 \%$, sendo semelhante aos 84 dias ( $>>0,05$ ), enquanto o maior valor de $22,62 \%$ foi observado para a idade de 112 dias de crescimento. Os valores de digestibilidade "in vitro" da matéria seca (DIVMS) variaram de 67,40\% a 57,40\% com o avanço da maturidade e apenas as idades de 84 e 112 dias foram semelhantes ( $>>0,05)$. Os valores de nitrogênio amoniacal por nitrogênio total $\left(\mathrm{N}-\mathrm{NH}_{3} / \mathrm{NT}\right)$ apresentaram diferença apenas para a idade de 28 dias com valor mais elevado que as demais $(\mathrm{p}<0,05)$. Foi observada diferença $(\mathrm{p}<0,05)$ apenas no $\mathrm{pH}$ da silagem do material colhido com 28 dias de crescimento, sendo superior aos demais com valor de 5,45. A Brachiaria brizantha apresenta potencial para produção de silagem com altas produções de matéria seca, entretanto, em idades jovens o baixo teor de MS da planta prejudica o processo fermentativo. O corte aos 68 dias de crescimento se apresenta como melhor opção para esta forrageira, uma vez que concilia alta produção de MS com bom valor nutritivo e características desejáveis para confecção de uma boa silagem.
\end{abstract}

Termos para indexação: Nitrogênio amoniacal, $\mathrm{pH}$, produção, valor nutritivo.

\begin{abstract}
The aim of this work was to determine the harvest moment of Brachiaria brizantha for silage production. The silages were opened at 56 days of fermentation. The treatments used were four growth ages $(28,56,84$, and 112 days). The experimental procedure followed a completely randomized design with four replications for each treatment. The dry matter (DM) production increased with the advance of the plant maturity, varying from 3.04 to $11.47 \mathrm{t} / \mathrm{ha}$. The lower dry matter value among the ensilages was observed for the 28-day treatment (17.16\%), which was similar to the 84-day treatment ( $>0.05$ ), while the highest value of $22.62 \%$ was observed for the age of 112 days of growth. The values of in vitro dry matter digestibility (IVDMD) in the silages varied from $67.40 \%$ to $57.40 \%$ with the advance of the maturity and were similar only $(\mathrm{p}<0.05)$ between the ages of 84 and 112 days. The values of ammoniacal nitrogen for total nitrogen $(\mathrm{N}-\mathrm{NH} 3 / \mathrm{NT})$ were higher at the age of 28 days than the other ages $(\mathrm{p}<0.05)$. The $\mathrm{pH}$ of the analyzed silages varied from 4.87 to 5.45 among the evaluated ages. The Brachiaria brizantha presents potential for silage production with high productions of dry matter. However, at younger ages, the low percentage of dry matter of the plant impairs the fermentation process. The harvest at 68 days of growth presents the better option for this plant, since it conciliates high production of DM with good nutritional value and desirable characteristic for a good silage confection.
\end{abstract}

Index terms: Ammoniacal nitrogen, nutritional value, $\mathrm{pH}$, production.

(Recebido em 17 de outubro de 2006 e aprovado em 27 de setembro de 2007)

\section{INTRODUÇÃO}

As gramíneas forrageiras tropicais apresentam limitações qualitativas que podem comprometer o desempenho por animal e por hectare. Além disso, a estacionalidade de produção, gera limitações na forragem ofertada aos animais.

${ }^{1}$ Médico Veterinário, Doutor em Zootecnia, Professor - Departamento de Zootecnia - Instituto Federal de Educação, Ciência e Tecnologia Sudeste de Minas Gerais, Campus Rio Pomba- IFET/Rio Pomba - Av. Dr. José Sebastião da Paixão, s/n, Bairro Lindo Vale - Rio Pomba, MG - 36.180-000 cgjayme@gmail.com

2Médico Veterinário, Doutor em Ciência Animal, Professor - Departamento de Clínica e Cirurgia Veterinárias/DCCV - Escola Veterinária/EV Universidade Federal de Minas Gerais/UFMG - Avenida Antônio Carlos, 6627 - Cx. P. 567 - 30123-970 - Belo Horizonte,MG - Imolina@vet.ufmg.br ${ }^{3}$ Engenheiro Agrônomo, Doutor em Zootecnia, Professor - Departamento de Zootecnia/DZOO - Escola Veterinária/EV - Universidade Federal de Minas Gerais/UFMG - Avenida Antônio Carlos, 6627 - Cx. P. 567 - 30123-970 - Belo Horizonte,MG - luciocg@vet.ufmg.br

${ }^{4}$ Médico Veterinário, Doutor em Zootecnia, Professor - Departamento de Zootecnia - Centro Federal de Educação Tecnológica de Uberaba/CEFETUBERABA - Rua João Batista Ribeiro, 4000 - Parque das Américas - 30430-390 - Uberaba, MG - diogogj@gmail.com

${ }^{5}$ Médico Veterinário, Doutor em Zootencia, Professor - Departamento de Ciências Agrárias/DCA - Universidade Estadual de Montes Claros/UNIMONTES Avenida Reinaldo Viana, 2630 - Bico da Pedra - Cx. P. 91 - 39440-000 - Janaúba, MG - depto.agrarias@unimontes.br

'Zootecnista, Doutor em Ciência Animal - Departamento de Zootecnia/DZOO - Escola Veterinária/EV - Universidade Federal de Minas Gerais/UFMG Avenida Antônio Carlos, 6627 - Cx. P. 567 - 30123-970 - Belo Horizonte, MG - iranborges@ufmg.br 
Para melhor equacionar o problema da disponibilidade de forragem ao longo do ano, uma das estratégias mais viáveis é a ensilagem do excedente produzido no período chuvoso, pois, além de fornecer volumoso para o período da seca, permite racionalizar o manejo intensivo das pastagens durante as águas (CORRÊA \& POTT, 2001).

A silagem de gramíneas tropicais é indicada principalmente para regiões sem aptidão agrícola, podendo ser uma boa alternativa para aumentar o estoque de forragem para seca, particularmente para categorias menos exigentes, como animais de cria e de recria, ou para regiões que disponham de concentrados baratos. A possibilidade de mais de um corte/ano e posterior aproveitamento do rebrote para pastejo podem compensar as dificuldades encontradas na confecção das silagens de capins (SILVA, 2001).

$\mathrm{O}$ alto teor de umidade e baixo teor de carboidratos solúveis no momento do corte das gramíneas tropicais, são fatores que inibem um adequado processo fermentativo, dificultando a confecção de silagens de boa qualidade (GALAN \& NÚSSIO, 2000; LAVEZZO, 1992; MCDONALD et al., 1991). Estes fatores influem negativamente sobre o processo fermentativo, impedindo o rápido decréscimo do $\mathrm{pH}$ a níveis adequados $(3,8$ a 4,2) e permitindo assim fermentações secundárias indesejáveis (WOOLFORD, 1984, citado por LAVEZZO, 1992).

O baixo valor nutritivo das forragens ensiladas em estádios avançados de desenvolvimento também interfere negativamente na qualidade do produto final ensilado (MORAIS, 1999).

O corte das plantas forrageiras destinadas a ensilagem deve ser feito no estádio vegetativo em que a planta encontra-se no seu "ponto de equilíbrio" entre produção de matéria seca e qualidade nutricional (PEREIRA \& REIS, 2001).

Neste trabalho, objetivou-se determinar o momento de colheita da Brachiaria brizantha produção de silagem.

\section{MATERIAL E MÉTODOS}

O trabalho foi conduzido na Fazenda Experimental Prof. Hélio Barbosa da Escola de Veterinária da UFMG, situada no município de Igarapé-MG. Foi utilizada uma área de Brachiaria brizantha já estabelecida em latossolo vermelho escuro. Com base na análise química do solo, no início do período chuvoso foi feita correção da acidez, aplicando $1500 \mathrm{Kg} / \mathrm{ha}$ de calcário dolomítico (PRNT: 63\%). Trinta dias depois, a pastagem passou por um corte de uniformização em 27 de outubro com roçadeira a $20 \mathrm{~cm}$ do nível do solo e recebeu adubação com $200 \mathrm{Kg}$ de uréia, $100 \mathrm{Kg}$ de cloreto de potássio e $300 \mathrm{Kg}$ de superfosfato simples. A área de $1024 \mathrm{~m}^{2}$ foi dividida em 16 canteiros de $64 \mathrm{~m}^{2}(8 \mathrm{x} 8 \mathrm{~m})$ dispostos num delineamento inteiramente casualisado. $\mathrm{O}$ capim foi submetido ao corte nas seguintes idades de crescimento: 28, 56, 84 e 112 dias, com quatro repetições (canteiros) por tratamento. $\mathrm{O}$ primeiro corte foi feito em 24 de novembro de 2001 e os demais a cada 28 dias.

Nas idades determinadas, a Brachiaria brizantha foi colhida a $20 \mathrm{~cm}$ do nível do solo, utilizando-se roçadeira costal. Para determinação da produção foi considerada uma área de $4 \mathrm{~m}^{2}(2 \times 2 \mathrm{~m})$ em cada canteiro e o material coletado foi pesado e, posteriormente, seco para determinação de sua matéria seca e estimativa da produção de MS (PMS). O restante do material coletado foi levado para um galpão, onde uma parte foi picada, em picadeira estacionária, em partículas de 10 a 30mm e ensilado. Para a ensilagem, foram utilizados 32 silos de laboratório confeccionados de tubos de PVC, com $40 \mathrm{~cm}$ de comprimento e $10 \mathrm{~cm}$ de diâmetro. A forragem foi prensada nos silos com soquete de madeira. Após ensilagem, os silos foram vedados com tampas de PVC providas de válvulas tipo Bunsen e lacrados com fita crepe.

Os silos foram levados para o Laboratório de Nutrição Animal da Escola de Veterinária da UFMG, foram mantidos à temperatura ambiente e abertos após 56 dias de fermentação. $\mathrm{Na}$ abertura dos silos, o material foi dividido em duas partes. De parte da amostra foram extraídos aproximadamente 200 $\mathrm{mL}$ de suco da silagem com auxílio de uma prensa hidráulica "Carver" modelo "C", onde foram determinados os valores de $\mathrm{pH}$ em potenciômetro Beckman Expandomatic SS-2 e nitrogênio amoniacal, determinado pelo método da destilação com óxido de magnésio e cloreto de cálcio, usando solução receptora de ácido bórico e titulação com ácido clorídrico a 0,01N segundo AOAC (1980). A outra parte do material foi acondicionado em bandejas de alumínio que foram pesadas e colocadas em estufa ventilada $\left(60 / 65^{\circ} \mathrm{C}\right)$ por 72 horas para pré-secagem. Depois de retirado da estufa, o material foi novamente pesado para determinação da matéria pré-seca. As amostras pré-secas foram moídas em moinho do tipo Willey, passadas em peneira de $1 \mathrm{~mm}$, e guardadas em recipientes de polietileno para análises posteriores. Foram determinados os teores de matéria seca a $105^{\circ} \mathrm{C}$ e a digestibilidade in vitro da matéria seca segundo Tilley \& Terry (1963).

As médias foram comparadas utilizando-se o teste SNK ("Student Newman Keuls") a 5\% de probabilidade. Foram testadas equações de regressão para PMS e DIVMS. 


\section{RESULTADOS E DISCUSSÃO}

Os resultados referentes à produção e teores de MS das silagens de Brachiaria brizantha estão apresentados na tabela 1. As produções de MS aumentaram com o avanço da idade da planta, variando de 3,04 a 11,47 t/ha para as idades de 28 e 112 dias.

$\mathrm{O}$ menor teor de MS entre as silagens foi observado aos 28 dias, $17,16 \%$, este valor foi semelhante ao encontrado aos 84 dias ( $p>0,05)$. Os demais valores foram diferentes entre si $(\mathrm{p}<0,05)$, sendo o maior valor de 22,62\% para a idade de 112 dias de crescimento. Foi observada correlação positivas do teor de MS com produção de MS $(\mathrm{r}=0,78 ; \mathrm{p}<0,01)$.

McDonald et al. (1991) indicam que para adequada fermentação no silo é necessário teor de MS acima de $20 \%$. Esta, por sua vez, é uma limitação na utilização das forrageiras tropicais para produção de silagem já que, quando apresentam adequado teor de MS, possuem baixo valor nutritivo.

Aumento na produção de MS com avanço da idade da planta foi observado no trabalho de Borges et al. (2002) que avaliando a Brachiaria brizantha cv. Marandu com adubação de $200 \mathrm{Kg}$ de fertilizante comercial 04-30-16 (N-P-K), encontraram produções de 0,$26 ; 1,78 ; 4,90$ e 3,18 t/ha aos $28,56,84$ e 112 dias respectivamente. Também Ruggieri et al. (1994) encontraram variação da ordem de 4,46 e 9,33 t/ha, respectivamente para as idades 28 e 56 dias. As variações existentes entre este e os demais experimentos devem-se à diferenças como fertilidade de solo, precipitação pluviométrica, adubação, temperatura, luminosidade, etc.

Os teores de DIVMS das silagens aparecem na tabela 2. O maior valor de DIVMS foi de $67,40 \%$ aos 28 dias de crescimento e o menor valor de $57,40 \%$ aos 112 dias. As idades de 56 e 84 dias foram semelhantes (p>0,05) com valores de $61,45 \%$ e $60,26 \%$, respectivamente. De acordo com McDonald et al. (1991), a redução na DIVMS com o avanço da maturidade da planta é decorrente do aumento de carboidratos estruturais, que possuem menor digestibilidade que as frações solúveis. Gerdes et al. (2000) encontraram valores de digestibilidade "in vitro" da Brachiaria brizantha colhida com 35 dias de $65,58 \%$ e $62,10 \%$ no verão e inverno, respectivamente.

Os teores de nitrogênio amoniacal $\left(\mathrm{N}-\mathrm{NH}_{3} / \mathrm{NT}\right)$ e $\mathrm{pH}$ das silagens estão apresentados na tabela 2. Os valores de $\mathrm{N}-\mathrm{NH}_{3} / \mathrm{NT}$ oscilaram de 3,29\% com 56 dias de crescimento a $14,38 \%$ com 28 dias, com média $6,82 \%$. Os valores não diferiram entre as idades $(\mathrm{p}>0,05)$, exceto para a idade de 28 dias que apresentou valor mais elevado que os demais $(\mathrm{p}<0,05)$. A fração protéica das silagens com alta umidade é, geralmente, extensivamente fermentada, ocasionando aumento do nitrogênio não protéico (SOEST, 1994). A graduação dos teores de nitrogênio amoniacal das silagens pode ser utilizada como indicativo da qualidade do processo de fermentação. Em geral, valores acima de $10 \%$ indicam redução de eficiência deste processo (TOMICH et al., 2003).

$\mathrm{O}$ pH das silagens variou de 4,87 a 5,45; com média 5,03 . Foi observada diferença $(\mathrm{p}<0,05)$ apenas no $\mathrm{pH}$ da silagem do material colhido com 28 dias de crescimento, sendo superior aos demais. $\mathrm{O}$ pH das silagens apresentou correlação positiva com os teores de nitrogênio amoniacal $(r=0,75 ; p<0,01)$ e correlação negativa com os teores de MS ( $r=-0,45 ; \mathrm{p}<0,03)$, indicando que altos valores de $\mathrm{pH}$ e baixos teores de MS contribuem para o aumento do nitrogênio amoniacal, conforme ocorreu na idade de corte de 28 dias. Segundo McDonald et al. (1991) e Soest (1994), silagens com alto $\mathrm{pH}$ e baixo conteúdo de MS, favorecem a quebra de proteínas em consequiência da ocorrência de fermentações secundárias por clostrídios e enterobactérias com desenvolvimento de aminas e ácido butírico, podendo ocorrer aumento nos teores de nitrogênio amoniacal de até três vezes.

Tabela 1 - Produção e teor de matéria seca (MS) do capim-Marandu colhido em quatro idades de crescimento.

\begin{tabular}{ccc}
\hline Tratamento (dias) & Produção MS (t/ha) & Teor MS (\%) \\
\hline 28 & $3,04^{\mathrm{D}}$ & $17,16^{\mathrm{C}}$ \\
56 & $4,37^{\mathrm{C}}$ & $18,83^{\mathrm{B}}$ \\
84 & $8,29^{\mathrm{B}}$ & $17,76^{\mathrm{C}}$ \\
112 & $11,47^{\mathrm{A}}$ & $22,62^{\mathrm{A}}$ \\
\hline Média geral & 6,79 & 19,09 \\
CV $(\%)$ & 10,16 & 3,52 \\
\hline
\end{tabular}

Médias na coluna seguidas de mesmas letras não diferem entre si pelo teste $\mathrm{SNK}, \mathrm{p}<0,05$. 
Valores elevados de $\mathrm{pH}$ têm sido observados em silagens de forrageiras tropicais. Mari (2003) verificou para as silagens de Brachiaria brizantha colhida com 30 e 90 dias valores de $\mathrm{pH}$ de 4,9 e 4,7 com teores de MS de 19,6\% e $20,7 \%$ respectivamente.

Para se determinar o momento de colheita de forrageiras para ensilagem, deve-se levar em consideração seu valor nutritivo, o processo fermentativo e produção de matéria seca por hectare. Tomich et al. (2003), propuseram um sistema de avaliação da fermentação de silagens baseado no valor de $\mathrm{pH}$ associado ao teor de matéria seca e avaliação do teor de nitrogênio amoniacal (Tabelas 3 e 4), além dos teores de ácido acético e butírico. Neste sistema, a classificação final das silagens é dada em uma escala de 0 a 100 pontos pela soma dos valores de cada tabela.

Quando analisa-se o processo fermentativo das silagens através do modelo proposto por Tomich et al. (2003) para os valores de pH e nitrogênio amoniacal, observa-se que do total de 50 pontos possíveis, a silagem confeccionada com capim colhido aos 28 dias teria pontuação igual a quinze pontos, enquanto os materiais colhidos nas idades de 56 e 84 dias teriam pontuação igual a 25 pontos. Já a silagem do material colhido aos 112 dias receberia 30 pontos. Estes dados demonstram que as silagens confeccionadas com Brachiaria brizantha colhida aos 56 e 84 dias apresentam classificação intermediária e que a melhor classificação quanto ao processo fermentativo é para a silagem do material colhido aos 112 dias. Porém, com o avanço da idade da planta, ocorre redução da digestibilidade, indicando que as forrageiras mais jovens possuem maior valor nutritivo. No entanto, quando as produções de matéria seca são analisadas, nota-se que ocorre o inverso com vantagem para as maiores idades. Para equacionar este problema foi proposta a determinação do momento de colheita que concilie produção de matéria seca e valor nutritivo, representado pela digestibilidade "in vitro" da matéria seca da silagem. A Figura 1 apresenta as curvas de produção de matéria seca da Brachiaria brizantha e de digestibilidade "in vitro" da matéria seca das silagens em finçaõ das idades de corte. De acordo com o gráfico o ponto de equilíbrio entre a produção de MS e DIVMS das silagens de Brachiaria brizantha dentro da faixa estudada neste experimento ocorreu próximo aos 68 dias de crescimento, onde a forrageira apresentou produção de MS e DIVMS de $6010,55 \mathrm{Kg}$ e $58,83 \%$, respectivamente.

Tabela 2 - Teores de digestibilidade "in vitro" da matéria seca (DIVMS), nitrogênio amoniacal $\left(\mathrm{N}_{-} \mathrm{NH}_{3} / \mathrm{NT}\right)$ e $\mathrm{pH}$ e das silagens de Brachiaria brizantha colhida em quatro idades de crescimento.

\begin{tabular}{cccc}
\hline Tratamento (dias) & Teor de DIVMS (\% MS) & ${\mathrm{N}-\mathrm{NH}_{3} / \mathrm{NT}}^{\mathrm{pH}}$ \\
\hline 28 & $67,40^{\mathrm{A}}$ & $14,38^{\mathrm{A}}$ & $5,45^{\mathrm{A}}$ \\
56 & $61,45^{\mathrm{B}}$ & $3,29^{\mathrm{B}}$ & $4,92^{\mathrm{B}}$ \\
84 & $60,26^{\mathrm{B}}$ & $5,94^{\mathrm{B}}$ & $4,87^{\mathrm{B}}$ \\
112 & $57,40^{\mathrm{C}}$ & $3,64^{\mathrm{B}}$ & $4,87^{\mathrm{B}}$ \\
\hline Média geral & 61,62 & 6,82 & 5,03 \\
CV (\%) & 2,48 & 23,77 & 4,44 \\
\hline
\end{tabular}

Médias na coluna seguidas de mesmas letras não diferem entre si pelo teste SNK, $p<0,05$.

Tabela 3 - Qualificação da fermentação da silagem em relação ao valor de pH associado ao conteúdo de matéria seca (MS).

\begin{tabular}{cccccc}
\hline & \multicolumn{5}{c}{ Conteúdo de MS (\%) } \\
\cline { 2 - 6 } & $<20$ & $20-30$ & $30-40$ & $>40$ & Pontuação \\
\cline { 2 - 6 } & $=4$ & $=4,2$ & $=4,4$ & 25 \\
$\frac{\pi}{2}$ & $>4,0-4,2$ & $>4,2-4,4$ & $>4,4-4,6$ & $>4,6-4,8$ & 20 \\
$\frac{0}{0}$ & $>4,2-4,4$ & $>4,4-4,6$ & $>4,6-4,8$ & $>4,8-5,0$ & 15 \\
$\frac{0}{\pi}$ & $>4,4-4,6$ & $>4,6-4,8$ & $>4,8-5,0$ & $>5,0-5,2$ & 10 \\
$>$ & $>4,6-4,8$ & $>4,8-5,0$ & $>5,0-5,2$ & $>5,2-5,4$ & 5 \\
& $>4,8$ & $>5,0$ & $>5,2$ & $>5,4$ & 0 \\
\hline
\end{tabular}

Fonte: Tomich et al. (2003). 
Tabela 4 - Qualificação da fermentação da silagem em relação ao conteúdo de nitrogênio amoniacal como proporção do nitrogênio total $\left(\mathrm{N}-\mathrm{NH}_{3} / \mathrm{NT}\right)$.

\begin{tabular}{cc}
\hline $\mathrm{N}^{-N_{N}} / \mathrm{NT}(\%)$ & Pontuação \\
\hline$<10$ & 25 \\
$10,0-13,0$ & 20 \\
$>13,0-17,0$ & 15 \\
$>17,0-21,0$ & 10 \\
$>21,0-25,0$ & 5 \\
$>25,0$ & 0 \\
\hline
\end{tabular}

Fonte: Tomich et al. (2003).

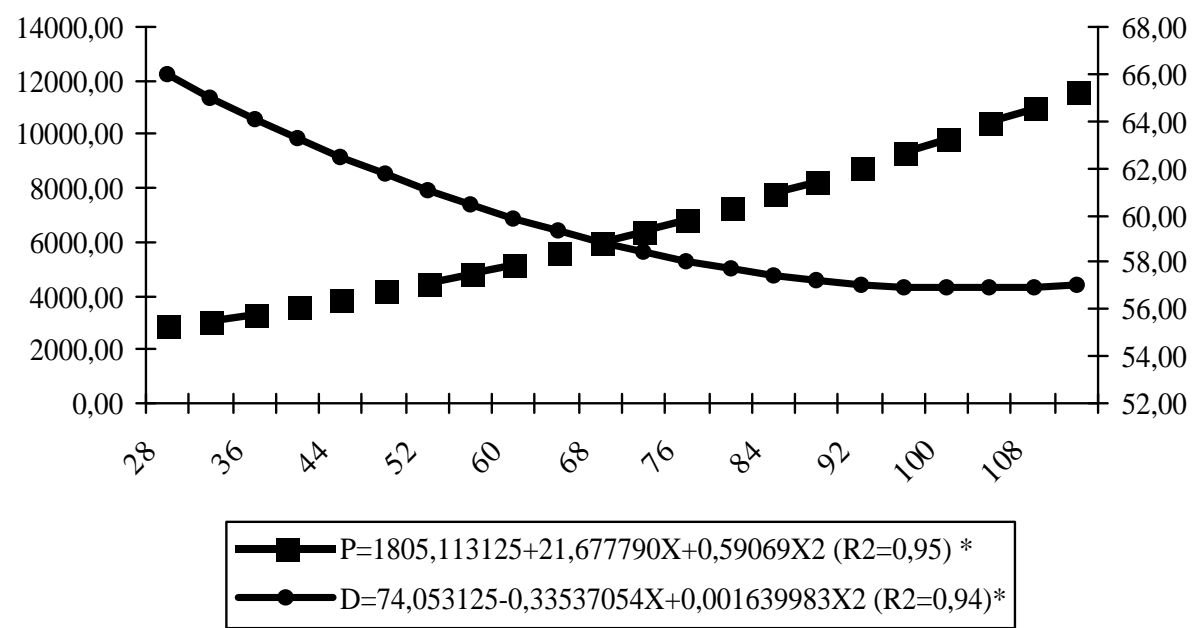

Figura 1 - Curvas de produção de matéria seca ( $\left(\begin{array}{l}1 \\ 1\end{array}\right)$ da Brachiaria brizantha e digestibilidade "in vitro" da matéria seca $(\bullet)$ das silagens de Brachiaria brizantha em função das idades de corte. * é significativo a $1 \%$ pelo teste SNK.

\section{CONCLUSÃO}

O capim Marandu apresenta altas produções de matéria seca e tem potencial para a produção de silagem. Entretanto, em idades mais jovens, o baixo teor de matéria seca da planta compromete o processo de fermentação.

Buscando conciliar o valor nutritivo com a produção de matéria seca da Brachiaria brizantha, a colheita da forrageira com idade próxima aos 68 dias de idade, apresenta-se como o melhor momento para a ensilagem.

\section{AGRADECIMENTOS}

Ao Conselho Nacional de Desenvolvimento Científico e Tecnológico - CNPq e à Fundação de Amparo à Pesquisa do Estado de Minas Gerais FAPEMIG, pelo apoio concedido.

\section{REFERÊNCIAS BIBLIOGRÁFICAS}

ASSOCIATION OFFICIAL ANALYTICAL CHENISTS. Official methods of analysis. 13. ed. Washington, DC, 1980. $1015 \mathrm{p}$.

BORGES, A. L. C.; RABELO, L. S.; GONÇALVES, L. C.; JAYME, C. G.; BORGES, I.; CAMPOS, E. F.; FERREIRA, J. J. C.; RODRIGUEZ, N. M.; SALIBA, E. O. S. Avaliação da Brachiaria brizantha cv. Marandu em oito idades de corte em Igarapé - MG: teores de matéria seca, produção de matéria seca e relação folha/haste. In: REUNIÃO ANUAL DA SOCIEDADE BRASILEIRA DE ZOOTECNIA, 39., 2002, Recife. Anais... Recife: SBZ, 2002.

CORRÊA, L. A.; POTT, E. B. Silagem de capim. In: SIMPÓSIO DE FORRAGICULTURA E PASTAGENS, 2., 2001, Lavras. Anais... Lavras: UFLA, 2001. p. 339-362. 
GALAN, V. B.; NUSSIO, L. G. Alimentos volumosos para o inverno: opções para viabilizar a produção de leite. In: SIMPÓSIO DE FORRAGICULTURA E PASTAGENS, 1., 2000, Lavras. Anais... Lavras: UFLA, 2000. p. 357-369.

GERDES, L.; WERNER, J. C.; COLOZZA, M. T.; POSSENTI, R. A.; SCHAMAMASS, E. A. Avaliação de características de valor nutritivo das gramíneas forrageiras Marandu, Setária e Tanzânia nas estações do ano. Revista Brasileira de Zootecnia, Viçosa, v. 29, n. 4, p. $955-963,2000$.

LAVEZZO, W. Ensilagem do capim-elefante. In: SIMPÓSIO SOBRE MANEJO DA PASTAGEM, 10., 1992, Piracicaba. Anais... Piracicaba: FEALQ, 1992. p. 169-275.

MARI, L. J. Intervalo entre cortes em capim Marandu (Brachiaria brizantha (Hochst. ex A. Rich.) Stapf cv. Marandu): produção, valor nutritivo e perdas associadas à fermentação da silagem. 2003. $138 \mathrm{f}$. Dissertação (Mestrado em Agronomia) - Escola Superior de Agricultura Luiz de Queiroz, Piracicaba, 2003.

MCDONALD, P.; HENDERSON, A. R.; HERON, S. J. E. The biochemistry of the silage. 2. ed. Marlow: Chalcombe, 1991. 340 p.
MORAIS, J. P. G. Silagem de gramíneas tropicais. In: SIMPÓSIO SOBRE NUTRIÇÃO DE BOVINOS, 7., 1999, Piracicaba. Anais... Piracicaba: FEALQ, 1999. p. 89-96.

PEREIRA, J. R. A.; REIS, R. A. Produção e utilização de forragem pré-secada. In: SIMPÓSIO DE

FORRAGICULTURA E PASTAGENS, 2., 2001, Lavras, MG. Anais... Lavras: UFLA, 2001. p. 311-338.

RUGGIERI, A. C.; FAVORETTO, V.; MALHEIROS, E. B.

Características de crescimento e produção de matéria seca da Brachiaria brizantha (HOCHST) STAPF. Cv. Marandu em função de níveis de nitrogênio e regimes de corte. Boletim da Indústria Animal, Nova Odessa, v. 51, n. 2, p. 149-155, 1994.

SILVA, J. M. Silagem de forrageiras tropicais. Brasília, DF: Embrapa, 2001. (Documentos, 51).

SOEST, P. J. van. Nutritional ecology of the ruminant. Ithaca: Comstock, 1994. 476 p.

TILLEY, J. M. A.; TERRY, R. A. A two-stage technique for the "in vitro" digestion of forage crops. Journal of British Grassland Society, London, v. 18, n. 2, p. 104-111, 1963.

TOMICH, T. R.; PEREIRA, L. G. R.; GONÇALVES, L. C. Características químicas para avaliação do processo fermentativo de silagens: uma proposta para qualificação da fermentação. Brasília, DF: Embrapa, 2003. (Documentos, 57). 